\title{
Gq/11-Induced and Spontaneous Waves of Coordinated Network Activation in Developing Frontal Cortex
}

\author{
D. Paola Calderon, Natalya Leverkova, ${ }^{*}$ and Alejandro Peinado \\ Department of Neuroscience, Albert Einstein College of Medicine, Bronx, New York 10461
}

\begin{abstract}
Repeated episodes of spontaneous large-scale neuronal bursting and calcium influx in the developing brain can potentially affect such fundamental processes as circuit formation and gene expression. Between postnatal day 3 (P3) and P7, the immature cortex can express one such form of activation whereby a wave of neuronal activity propagates through cortical networks, generating massive calcium influx. We previously showed that this activity could be triggered by brief stimulation of muscarinic receptors. Here, we show, by monitoring large cortical areas at low magnification, that although all areas respond to muscarinic agonists to some extent, only some areas are likely to generate the coordinated wave-like activation. The waves can be triggered repeatedly in frontal areas where, as we also show, waves occur spontaneously at a low frequency. In parietal and occipital areas, no such waves are seen. This selectivity may be related in part to differences in the cortical distribution of dopaminergic signaling, because we find that activation of dopamine receptors enables the response. Because M1 muscarinic receptors are typically coupled with G- $\alpha$ q/11, we investigated whether other receptors known to couple with this G-protein (group I glutamate metabotropic receptors, neurotensin type 1) could similarly elicit wave-like activation in responsive cortical areas. Our results suggest that multiple neurotransmitter systems can enable this form of activation in the frontal cortex. The findings suggest that a poorly recognized, developmentally regulated form of strong network activation found predominantly in the frontal cortex could potentially exert a profound influence on brain development.
\end{abstract}

Key words: calcium (Ca); cortex; development; dopamine; frontal; muscarinic; neurotensin; mGluR5; spontaneous

\section{Introduction}

The remarkably complex orchestration of nervous system development requires the influence of activity at many different stages (Katz and Shatz, 1996; Zhang and Poo, 2001). Patterned activity driven by external stimuli is crucial in shaping connectivity once the basic patterns have been established. At earlier times, however, both before and during the initial stages of synapse formation, many neural structures are capable of generating their own activity spontaneously (for review, see Feller, 1999; O'Donovan, 1999; Wong, 1999; Ben-Ari, 2001; Zhang and Poo, 2001). The importance of this endogenous activity in brain development is beginning to be appreciated as evidence points to its role in regulating neuronal gene expression and guiding specific stages of circuit formation.

The occurrence of spontaneous bursts of activity during development has been demonstrated in diverse neural structures including those responsible for motor pattern generation and visual and auditory processing, as well as higher processing centers such as the hippocampus and entorhinal cortex (Meister et al., 1991; Yuste et al., 1992; Chub and O’Donovan, 1998; Garaschuk et al., 1998; Milner and Landmesser, 1999; Weliky and Katz,

Received July 10, 2004; revised Sept. 30, 2004; accepted Jan. 5, 2005

This work was supported by National Institutes of Health Grant NS39901 to A.P.

*N.L. contributed only to the experiments and analysis related to spontaneous activity.

Correspondence should be addressed to Dr. Alejandro Peinado, Kennedy Center, Room 522, Albert Einstein

College of Medicine, 1300 Morris Park Avenue, Bronx, NY 10461. E-mail: peinado@aecom.yu.edu.

D01:10.1523/JNEUROSCI.2765-04.2005

Copyright $\odot 2005$ Society for Neuroscience $\quad$ 0270-6474/05/251737-13\$15.00/0
1999; Leinekugel et al., 2002). In several instances, an instructive role for this spontaneous activity in normal development has been demonstrated. In the developing visual system, spontaneous episodes of retinal activity contribute to the process of axonal segregation into eye-specific layers in the lateral geniculate nucleus (Shatz, 1996; Penn et al., 1998; Stellwagen and Shatz, 2002); in the developing olfactory system, spontaneous activity is required for the formation and maintenance of olfactory maps $(\mathrm{Yu}$ et al., 2004); and spontaneous activity in the developing spinal cord contributes to the establishment and maintenance of transmitter phenotype (Spitzer et al., 1993; Gu and Spitzer, 1995; Watt et al., 2000; Borodinsky et al., 2004).

One region that has been poorly characterized with respect to spontaneous activity during development is the frontal cortex, a region that in the adult brain is associated with many higherorder behaviors and the development of which is perturbed in some forms of mental illness in humans. The frontal cortex is unique among cortical areas in its extensive interconnections with the rest of the brain (Ongur and Price, 2000; Vertes, 2004). Spontaneous activity in this region is therefore likely to affect many other brain regions. Here, we show that at early stages of postnatal development, a group of modulatory neurotransmitters, the receptors for which are not known to exhibit areaspecific expression, induce robust wave-like activation of frontal and other cortical areas. We also show that this form of activation can occur spontaneously, albeit infrequently, in brain slices. Our findings show that during a period when formation of new synapses is occurring at a fast pace (De Felipe et al., 1997) and when 
dramatic changes in patterns of gene expression are taking place (Stead et al., 2003), frontal cortical networks exhibit properties that render them prone to a form of coordinated activation involving large changes in neuronal $\left[\mathrm{Ca}^{2+}\right]_{i}$; under the same conditions, neurons located in the somatosensory and visual regions of the parietal and occipital cortex, respectively, exhibit smaller, mostly uncoordinated calcium transients.

\section{Materials and Methods}

Tissue preparation. The experiments described here were performed on 300- to $400-\mu \mathrm{m}$-thick slices obtained from Long-Evans and Wistar rat pups (no strain differences were observed) during the first week after birth. Pregnant dams were purchased from Charles River (Wilmington, MA) and were delivered to our animal facility on either the 5 th or the 8 th day of gestation. Slices were cut in ice-cold normal artificial CSF (ACSF) using a vibratome. The composition of the ACSF, modified according to MacGregor et al. (2001) to prevent neuronal swelling, was as follows (in mM): $109 \mathrm{NaCl}, 2.5 \mathrm{KCl}, 1.25 \mathrm{KH}_{2} \mathrm{PO}_{4}, 1 \mathrm{MgCl}-6 \mathrm{H}_{2} \mathrm{O}, 35 \mathrm{NaHCO}_{3}, 10$ glucose, $20 \mathrm{HEPES}$, and $2 \mathrm{CaCl}_{2}\left[1 \mathrm{mM} \mathrm{CaCl}_{2}\right.$, a more physiological concentration, and one that promotes the ability of neurons to exhibit sustained firing (Sanchez-Vives and McCormick, 2000), was used in experiments examining spontaneous activation]. The $\mathrm{pH}$ after equilibration with $95 \% \mathrm{O}_{2} / 5 \% \mathrm{CO}_{2}$ was 7.4 . The plane of section of the slices was coronal, sagittal, or horizontal. Coronal slices were obtained between the rostrocaudal landmarks of the anterior commissure and anterior hippocampus. Sagittal slices were taken from a region between 0.5 and 1.5 $\mathrm{mm}$ from the midline. Horizontal slices were obtained from the region immediately dorsal to the rhinal sulcus. All slices included the entire extent of the brain, except that the two hemispheres were often separated to reduce the size of the slice. Because cytoarchitectonic features are not well defined at these young ages, cortical areas were identified solely by their relative spatial location within the cortex, according to published maps (Zilles, 1985; Paxinos et al., 1991).

Fura-2 loading. Loading was performed by bath application of fura-2 acetoxymethyl ester (fura-2 AM; Molecular Probes, Eugene, OR) for $2 \mathrm{~h}$ at $30^{\circ} \mathrm{C}$ in ACSF containing $5 \mu \mathrm{g} / \mathrm{ml}$ fura- $2 \mathrm{AM}$. The indicator dye was added, from a stock prepared in DMSO $(50 \mu \mathrm{g}$ in $50 \mu \mathrm{l})$, to ACSF that had been filtered $(0.2 \mu \mathrm{m})$. The dye in the ACSF was then briefly sonicated before use. To ensure adequate oxygenation of the submerged slice during dye incubation, the loading chamber ( $4 \mathrm{~cm}$ diameter; $1 \mathrm{ml}$ volume of ACSF) was kept in a closed container that was oxygenated continuously with $95 \% \mathrm{O}_{2} / 5 \% \mathrm{CO}_{2}$ and kept on a rotating platform at $60 \mathrm{rpm}$.

Calcium imaging. For imaging, slices were removed from the loading solution and placed in standard oxygenated ACSF, transferred to a recording chamber on the stage of an upright compound microscope (Zeiss Axioskop, Fixed Stage, equipped with a $z$-axis motor drive; Nikon E600 physiostation), and perfused with oxygenated ACSF at a rate of 2-3 $\mathrm{ml} / \mathrm{min}$ in a temperature-controlled $\left(29 \pm 1^{\circ} \mathrm{C}\right.$ ) perfusion chamber (volume, 200-400 $\mu \mathrm{l}$; Warner Instruments, Hamden, CT). Slices were viewed with a $3.5 \times$ dry or $10 \times, 20 \times, 40 \times$, or $63 \times$ water-immersion objective. Epifluorescence imaging of fura-2 intensity was performed with a $75 \mathrm{~W}$ Xenon light source and a low-light CCD digital camera. One of two cooled CCD cameras was used: (1) $512 \times 512$ pixel Frame Transfer, $1 \mathrm{MHz}, 12$-bit (Roper Scientific, Princeton, NJ) and (2) $1024 \times 1280$ pixel, 12.5 MHz, 12-bit (SVGA Sensicam; Cooke Corp., Tonawanda, $\mathrm{NY}$ ). In most instances, a $0.5 \times$ adapter was placed in the trinocular port of the microscope so as to capture a larger field (up to $3 \times 2.4 \mathrm{~mm}$ ) with the CCD camera. Minimal exposure of the preparation to the excitation light was achieved by controlling a shutter in the excitation light path (Ludl Electronics, Hawthorne, NY), reducing light intensity with neutral density filters, and operating the camera on a $5 \times 5$ pixel-binning mode to increase sensitivity. All measurements of relative changes in $\left[\mathrm{Ca}^{2+}\right]_{i}$ were made at a single excitation wavelength using a $380 \pm 5 \mathrm{~nm}$ bandpass filter (Chroma Technology, Brattleboro, VT). Emission fluorescence was filtered with a $400 \mathrm{~nm}$ long-pass filter. In some instances, static images were also acquired at the isosbestic point (calcium-independent fluorescence) using a $360 \pm 5 \mathrm{~nm}$ excitation filter. Acquisition protocols consisted of 2- or 12-min-long time-lapse sequences ( 200 or $250 \mathrm{~ms}$ integra- tion times per frame) of fura-2 fluorescence, acquired using custom scripts written in IPLab software (Scanalytics, Fairfax, VA) running on Macintosh and Windows platforms.

Drug application. Pharmacological agents were applied by a multivalve, single-output gravity perfusion system (ALA Scientific, Westbury, NY). Ten seconds of baseline fura-2 fluorescence were recorded during perfusion of control ACSF, and then drug-containing ACSF was applied for $20 \mathrm{~s}$. A minimum intertrial time of $10 \mathrm{~min}$ in the presence of flowing control ACSF was used in all experiments to allow for washout of agonist.

The minimum perfusion time required to reach the desired agonist concentration in the bath was determined in separate experiments using a water-soluble fluorescent dye (NBD-methylglucamine; M.W. 356; Molecular Probes) dissolved in ACSF at typical agonist concentrations. The change in ACSF fluorescence as a function of time was measured at the chamber with the CCD camera using appropriate optical filters. A $20 \mathrm{~s}$ perfusion time was found to be necessary for the dye fluorescence in our bath to reach a steady-state level, and most agonist applications were therefore limited to $20 \mathrm{~s}$. Actual concentrations inside the slice were not measured but are presumed to be lower than in the bath.

Stock drug solutions were prepared at the indicated concentrations: $100 \mathrm{~mm}$ 1-aminocyclopentane-trans-1,3-dicarboxylic acid (ACPD; Tocris Cookson, Ellisville, MO); 25 mm muscarine chloride (Sigma, St. Louis, MO); 6.1 mм Neuromedin-N (Peninsula Laboratories, Belmont, CA); $6.1 \mathrm{~mm}$ neurotensin 8-13 (Peninsula Laboratories); $20 \mathrm{~mm}( \pm$ )-trans10,11-dihydroxy$5,6,6 \mathrm{a}, 7,8,12 \mathrm{~b}$-hexahydrobenzo[a]phenanthridine (dihydrexidine hydrochloride; Tocris Cookson); $20 \mathrm{~mm}(R)-(+)-7$-chloro-8-hydroxy-3-methyl-1phenyl-2,3,4,5-tetrahydro-1H-3-benzazepine hydrochloride (SCH23390; Tocris Cookson); 100 mм picrotoxin (Sigma); $50 \mathrm{~mm}(R S)$-1-aminoindan-1,5dicarboxylic acid (AIDA; Tocris Cookson).

Analysis of calcium imaging. As mentioned, measurements of changes in fura-2 fluorescence $\left(\Delta\left[\mathrm{Ca}^{2+}\right]_{\mathrm{i}}\right)$ as a function of time were done at a single wavelength, not by the wavelength ratio method. All analysis and processing, as well as playback of the image sequences for visual inspection, was made using IPLab software. Areas over groups of cells (low magnification) or individual cells (high magnification) were selected, and the mean intensity at each frame was measured. Raw data were in the form of a linear 12-bit intensity scale $(0-4096)$. Raw data were first plotted as fluorescence intensity versus time and subsequently converted, for comparison across experiments, to a relative scale $\left(\% \Delta F / F_{\text {baseline }}\right)$. The script written for conversion to a relative scale also inverted the polarity of the signal, thus converting negative changes in fura-2 fluorescence (reflecting increases in $\left[\mathrm{Ca}^{2+}\right]_{\mathrm{i}}$ ) to positive changes in $\% \Delta F / F$.

To illustrate the spatial changes in calcium resulting from agonist application, the raw sequences were processed to highlight changes in fluorescence intensity from baseline levels. This processing, referred to as 1 st $-\mathrm{N}$ th processing, involves making a new sequence in which each new frame is the result of subtracting all frames in the sequence from a "baseline" frame taken early in the sequence. In this new sequence, changes in $380 \mathrm{~nm}$ fluorescence are represented by the intensity of grayscale values; pixels that changed (decreased) the most appear whitest in the processed images. Image contrast was subsequently adjusted to further highlight the response differences between different cortical areas. It is important to point out that these images are used here for illustrating the spatial pattern of activation, not the intensity of this activation or the level of steady-state baseline fluorescence throughout the imaged area.

Traces representing the first derivative of the fura- 2 fluorescence (see Fig. 6) were obtained by processing an entire raw sequence of images with an algorithm that subtracted the value of each pixel in each frame from the value of the pixel five frames earlier. Negative values were filtered out during this subtraction by virtue of the fact that, in the image format used here, pixel values are represented as positive integers only. Because the fastest rate of change in $\left[\mathrm{Ca}^{2+}\right]_{\mathrm{i}}$ typically occurs at the beginning of a response, a positive peak in the derivative trace provides a useful measure of response onset and is a convenient way of displaying and comparing the timing of responses for individual cells within a field of view.

Quantification of fura-2 loading. To compare the extent of fura-2 AM loading in high and low responding areas, the digital camera with a larger image area was used (Sensicam SVGA). The $8.6 \times 6.9 \mathrm{~mm}$ sensor of this camera allowed analysis of a more extensive area on both sides of the 
wave propagation boundary than was possible with the other camera. In addition, for better cellular resolution, images were acquired using a $2 \times$ 2 pixel-binning mode instead of the usual $5 \times 5$ pixel-binning mode used during acquisition of long sequences. Finally, instead of limiting the capture to a single image at one focal plane, we acquired a series of images at equally spaced, short Z-plane intervals to include the largest possible number of labeled cells per field of view. The data from all of the images in a series were subsequently combined into a single frame using a maximum-brightness algorithm, and the background illumination was corrected with a rolling ball radius routine (IPLab; Scanalytics). On the resulting frame, two areas were defined after locating the boundary of wave propagation. Measurements of mean fluorescence intensity were obtained from each of these two areas. For this quantification, images were acquired at a $350 \mathrm{~nm}$ excitation wavelength, which is close to the isobestic (calcium-insensitive) wavelength of fura-2, to minimize differences attributable to baseline calcium concentration.

Nissl stain. Brain slices were fixed in $4 \%$ paraformaldehyde with picric acid in $0.1 \mathrm{~m}$ phosphate buffer for $2 \mathrm{~h}$, followed by an overnight cryoprotective incubation in fixative with $30 \%$ sucrose. Slices were subsequently resectioned at a thickness of $20 \mu \mathrm{m}$ in a cryostat, mounted on glass slides, and stained with a red fluorescent Nissl stain (NeuroTrace 530/615; Molecular Probes) according to the manufacturer's suggested protocol using a 1:200 dilution of the dye.

\section{Results}

A region in the mediodorsal cortex exhibits wave-like largescale neuronal activation

We have previously shown a novel form of wave-like network activity in postnatal day 3 (P3) to P7 cortical slices bulk-loaded with the calcium indicator fura- 2 and imaged at low magnification (Peinado, 2000). In that study, which was done exclusively on slices of whole brains cut in the coronal plane, we found that brief stimulation of muscarinic receptors produced a wave of neuronal calcium increase that spread through cortical networks. The signature of this event, when calcium-dependent fura-2 fluorescence is averaged over tens to hundreds of neurons, consists of two components (Fig. 1a). The initial response to agonist is a slow, low-amplitude increase in calcium, a reflection of the fact that neurons are responding in a diffuse, noncoordinated manner. Then, occurring with a variable delay and typically riding on the low-amplitude response, there is a fast, large-amplitude calcium increase. It is this second component, not the first, that can be seen to travel as a wave when a sequence of fura- 2 images is played back. We refer to the low-amplitude response as the prewave component, although it is not always associated with a wave. These two different response components are discussed further below. The electrophysiological correlate of the calcium wave, recorded with whole-cell current clamp, was shown to consist of a burst of action potentials giving rise to a plateau potential (Peinado, 2000). Another potential electrophysiological correlate of the calcium waves are the field potential oscillations in the $\beta$ frequency range recorded extracellularly by Kilb and Luhmann (2003) in response to muscarinic stimulation of the neonatal cortex. We note, however, that whereas the field oscillations are abolished by blockade of NMDA subtype receptors (Kilb and Luhmann, 2003), expression of muscarine-induced calcium waves does not require this receptor (Peinado, 2000).

One notable feature of the waves induced with muscarinic stimulation was that they were not observed consistently as one moved from one cortical site to another. At many sites, despite repeated attempts at inducing them with agonist, no calcium wave was produced. Instead, only the low-amplitude prewave component could be detected in response to stimulation. Here, we show that the type of response obtained depends on the region of the slice that is being imaged and that, as shown in Figure $1 a$,
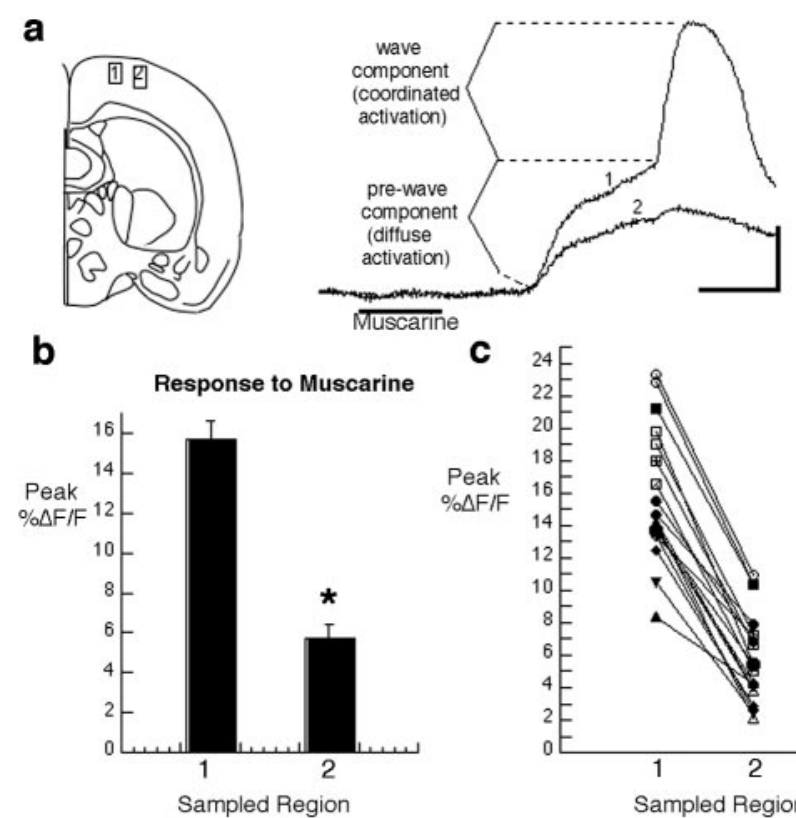

Figure 1. Activation of developing cortical networks by muscarinic stimulation is stronger in a dorsomedial region of coronal slices. $\boldsymbol{a}$, Schematic diagram of a coronal slice showing the location from which sample records at the right were obtained. The traces show the response to muscarine ( $25 \mu \mathrm{m}$ at the time shown) at each of two regions of interest separated by $200 \mu \mathrm{m}$ in a P4 slice. Each trace represents changes in calcium-dependent fura-2 fluorescence sampled at a region of interest that includes many cells. By moving the field of view progressively more lateral until the wave termination site was found, both types of responses could be examined simultaneously in adjacent regions. $\boldsymbol{b}$, Grouped data from results like the one shown in $\boldsymbol{a}$. Error bars represent values for the maximum percentage of change in fura-2 fluorescence from baseline to peak (mean \pm SEM; $n=19$ slices; P3-P7). ${ }^{*} p<0.0001$ (Student's $t$ test, paired data). c, Paired data for each individual experiment show that the difference between values is very consistent. Calibration: $20 \mathrm{~s}, 5 \% \Delta \mathrm{F} / \mathrm{F}$.

most of the calcium waves triggered by muscarinic stimulation in coronal slices taken from the middle third of the brain occur in an area of the cortex located dorsomedially but not in the more lateral cortex, where the somatosensory region is located. Thus, the large response to muscarine obtained in coronal slices typically consists of a wave of activation that initiates medially and spreads from medial to lateral across a segment of the cortex located in the dorsalmost region.

To try to understand why only a small region of the cortex was responding with waves of activity, we began by asking whether wave propagation ends abruptly or through a gradual diminution of the wave. When necessary, this was accomplished by repeatedly inducing waves and following their propagation through successively more lateral fields of view until a termination site could be seen and was approximately centered within the field of view. For each slice examined (ages P3-P7; $25 \mu \mathrm{m}$ muscarine bath applied for $20-30 \mathrm{~s}$ ), we measured the mean peak $\% \Delta F / F$ for two rectangular regions of interest, separated by $200 \mu \mathrm{m}$, on either side of the wave termination site (Fig. $1 a-c$ ). Records from 19 different slices were included in this analysis, the criterion for inclusion being that the site of wave termination was within the field of view. This ensured that measurements of calciumdependent changes in fura-2 fluorescence from both sides of the "boundary" could be obtained simultaneously (i.e., in response to the same episode of agonist stimulation). This analysis showed that as a result of the wave-like response, the spatially averaged calcium transients medial to the wave termination site were, on average, $>2.5$ times larger $(15.7 \pm 0.9 \% \Delta F / F$ at peak; $n=19$ 
slices) than responses in the adjacent, more lateral cortex $(5.7 \pm 0.6 \% \Delta F / F$ at peak; mean \pm SEM; same 19 slices). The paired data from each individual slice also showed that this difference occurred with remarkable consistency (Fig. 1c). Thus, the transition from wave to no wave occurs abruptly within a short cortical distance, suggestive of a fairly abrupt change in the properties of the neurons.

The difference in peak $\% \Delta F / F$ was primarily attributable to the absence of the fast calcium transient in the more lateral region. However, in some instances, the prewave component of the calcium response was smaller in the lateral cortex (Fig. 1a), and therefore this also contributed to the difference. In other instances, however, the prewave component in the region distal to the wave termination was the same or larger than the prewave component in the more medial cortex. This lack of correlation between the magnitude of the prewave component and the presence and absence of a wave suggests that an insufficient response to muscarinic stimulation is unlikely to be the explanation for the absence of waves in the more lateral cortex. Instead, it seems more likely that cortical areas that are able to generate a wave possess some additional property that specifically enables this response.

We next measured the extent of the dorsal cortical region capable of exhibiting wave-like activity. This analysis consisted of simply measuring the distance along the pial curvature for the cortical area encompassing the wave-competent region. The results, shown in Figure 2, are somewhat paradoxical. On the one hand, there can be much variability from slice to slice in the mediolateral extent of the wave competent area (range, 585-1575 $\mu \mathrm{m}$; mean \pm $\mathrm{SD}, 1093 \pm 304 ; n=13$; P3-P6) (Fig. $2 b$ ). On the other hand, not only is the termination itself abrupt, but the location of wave termination in any one slice is relatively constant from one agonist application to the next (Fig. 2c). An additional observation that may begin to explain this apparent contradiction is shown in Figure $2 d$. In this example, a single episode of agonist stimulation gave rise to two wave events closely spaced in time. Interestingly, the second wave can be seen to advance $200 \mu \mathrm{m}$ beyond the termination site of the first wave, suggesting that possibly the residual network excitation in the wake of the first wave is responsible for allowing the additional advance of the second wave. Together, these observations suggest that the factors underlying wave competence in the dorsal cortex may, to some extent, be dynamic and modifiable by activity rather than existing as an all-or-none property of the network with fixed and rigid boundaries. For example, it is possible that a key component(s) in the mechanism of wave generation and propagation is distributed in a gradient manner and that how far a wave travels down this decreasing gradient is, to some extent, dependent on previous activation history. b

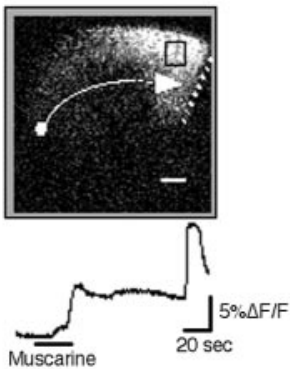

b

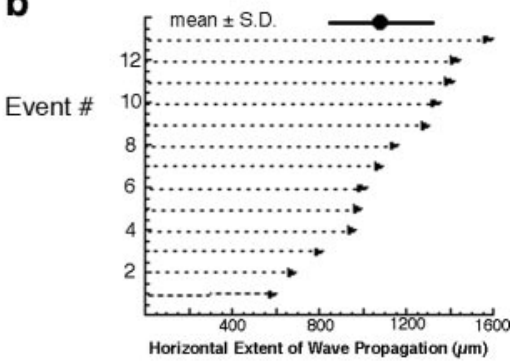

e

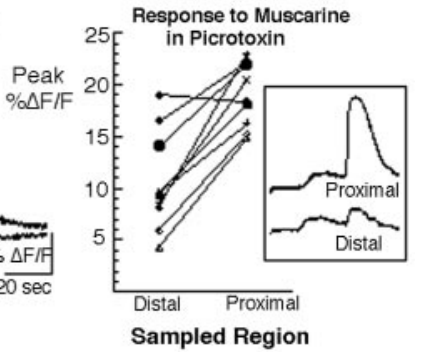

Sampled Region

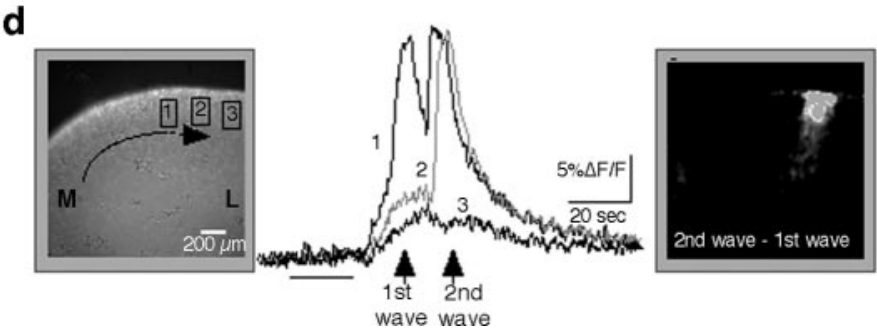

Figure 2. The distance over which waves propagate on the dorsal region of the cortex is variable. $\boldsymbol{a}$, Spatial extent of a response recorded in the dorsal region of a P5 coronal slice, in the area shown on the left (for a description of image processing algorithms, see Materials and Methods, Analysis of calcium imaging). The dot and arrow represent the approximate initiation site and direction ion, respectively, of waves in this region. The dotted line marks the boundary of wave propagation. The black square is a P4 slice. Traces show the activity at each of the three regions shown on the left image. M, Medial; L, lateral.e, Effect of blockade GABA $A_{A}$ receptor channels with picrotoxin $(100 \mu \mathrm{m})$ on the response to agonist shows that differences in amplitude between difference in responsiveness is preserved in picrotoxin. Mean \pm SEM of response at peak: $10.6 \pm 1.6$ (distal cortex) versus $18.9 \pm$ 1.0 (proximal cortex). $p<0.0004$ ( $t$ test, paired data).

One factor that may influence the boundary of wave propagation is the level of GABAergic inhibition. To examine its role in establishing the dichotomy between wave-competent and waveincompetent areas, we elicited waves in the presence of the $\mathrm{GABA}_{\mathrm{A}}$ channel blocker picrotoxin $\left[\mathrm{GABA}_{\mathrm{B}}\right.$ responses in the cortex do not appear until the end of the second postnatal week (Luhmann and Prince, 1991; Owens et al., 1996)]. The addition of picrotoxin resulted in larger amplitude responses in all cortical areas, indicative of the inhibitory nature of GABA in the cortex. Despite this general increase in all but one of nine experiments, the difference in signal amplitude between both sides of the termination site remained significant in the presence of picrotoxin (Fig. 2e). However, when considering the grouped data, the increase in signal amplitude as a result of adding picrotoxin was larger for the more lateral sites $(5.8 \pm 0.6 \% \Delta F / F$ vs $10.6 \pm$ $1.6 \% \Delta F / F ; p=0.002)$ than for the sites exhibiting the wave response $(15.7 \pm 0.9 \% \Delta F / F$ vs $18.9 \pm 1.0 \% \Delta F / F ; p=0.04)$, suggesting that although $\mathrm{GABA}_{\mathrm{A}}$ inhibition by itself is not defining the borders, GABA does have an inhibitory effect on network responses to muscarine during the first postnatal week, and this 
effect appears greater in regions of the cortex not invaded by the wave.

\section{No clear cytoarchitectonic boundaries are present at wave termination sites}

The observations described above raise the question of what accounts for the abrupt termination in the spread of this activity or, in other words, what determines that one region will respond with a strong calcium wave and a neighboring region will only respond with a low-amplitude calcium response. Are there, for example, clear cytoarchitectonic transitions at the site where waves terminate that might be indicative of boundaries between known functional areas? Fura-2 imaging done at an excitation wavelength of $350 \mathrm{~nm}$ (at which fluorescence emission has a very low calcium sensitivity) provides a good live "histological" stain with which to visualize differences in cell density and/or size. On the basis of this staining, we were unable to see any clear cytoarchitectonic transition at the boundary where the wave propagation ends ( $n=5$ coronal slices, dorsal region) (Fig. 3 ). We were also unable to detect any differences in overall dye loading between the two regions. Thus, despite very clear differences in the ability of neighboring areas to sustain wave activity (Fig. 3c), there was no significant difference in the staining level between those same regions (Fig. $3 d$ ). Likewise, when adjacent sections were stained with a fluorescent Nissl stain, we found no evidence of a cytoarchitectonic boundary that might correlate with the wave termination site. These results suggest that gross anatomical features of the cortex at these ages are not useful in identifying regions exhibiting calcium waves. Instead, less obvious and as yet unidentified features of the cortex such as underlying circuitry or the intrinsic physiological properties of cells in each region are more likely to correlate with the response type. The even distribution of fura- 2 staining seen at $350 \mathrm{~nm}$ also makes it unlikely that a technical artifact related to differential loading of dye is an explanation for the consistent absence of wave responses in some areas or its presence in others.

\section{Other $\mathrm{G}_{\mathrm{q} / 11}$-linked G-protein-coupled receptors mediate the} same activation as muscarinic receptors

Wave-like responses after muscarinic stimulation are mediated by M1 and M3 subtype receptors (Peinado, 2000), both of which couple with G- $\alpha_{\mathrm{q} / 11}$ and activate the phospholipase C pathway. Other neurotransmitter receptors capable of activating the same signaling pathway are widely expressed in the developing cortex. We were therefore interested in finding out whether the same type of differential activation of cortical areas can be mediated by other receptors. One such group of receptors abundantly expressed in the developing cortex is the glutamate metabotropic type (mGluR), of which group I (mGluR1 and mGluR5) is coupled to G- $\alpha_{\mathrm{q} / 11}$. Experiments in which muscarine was replaced by the mGluR agonist ACPD $(40 \mu \mathrm{M})$ have consistently shown that this agonist elicits the same phenomenon (Fig. 4a). The spatial distribution of the ACPD-induced response appeared indistinguishable from that induced by muscarine (data not shown). The response elicited by ACPD is blocked by the mGluR5 antagonist 2-methyl-6-(phenylethynyl)pyridine (MPEP; $2.5 \mu \mathrm{M} ; n=5$ ) (Fig. 4a). In contrast, AIDA, a group I mGluR antagonist that blocks primarily mGluR1 when used at 100-200 $\mu \mathrm{M}$ (Moroni et al., 1997), did not block the wave response to ACPD $(n=5)$ (Fig. $4 b)$. The group I mGluR agonist $(R S)$-3,5-dihydroxyphenylglycine $(20 \mu \mathrm{M})$ mimics the ACPD effect (data not shown).

Glutamate is a more abundant neurotransmitter in the cortex than acetylcholine. Having shown that mGluR5 can mediate net-

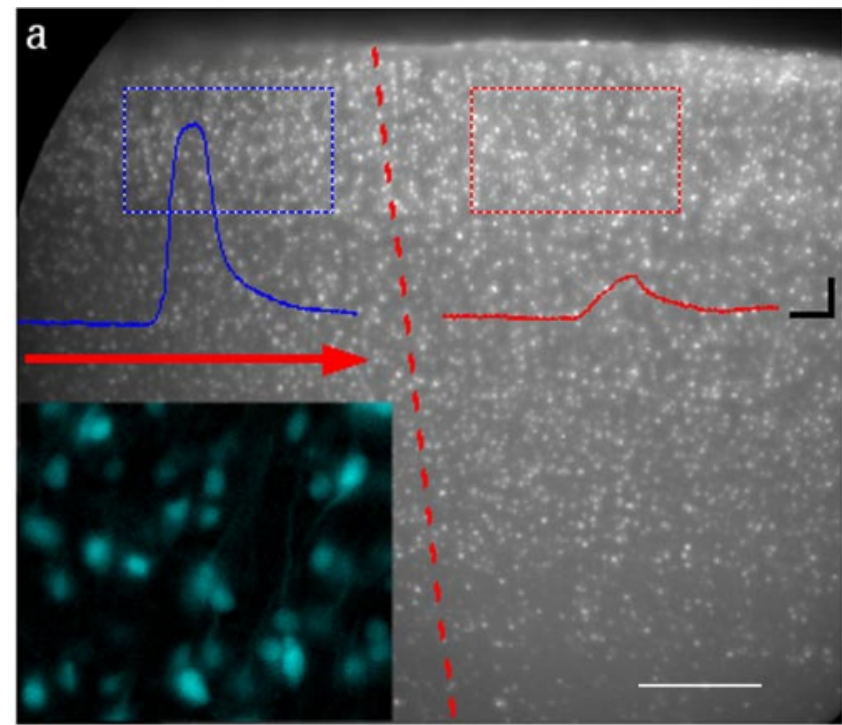

b

$\mathrm{C}$

d
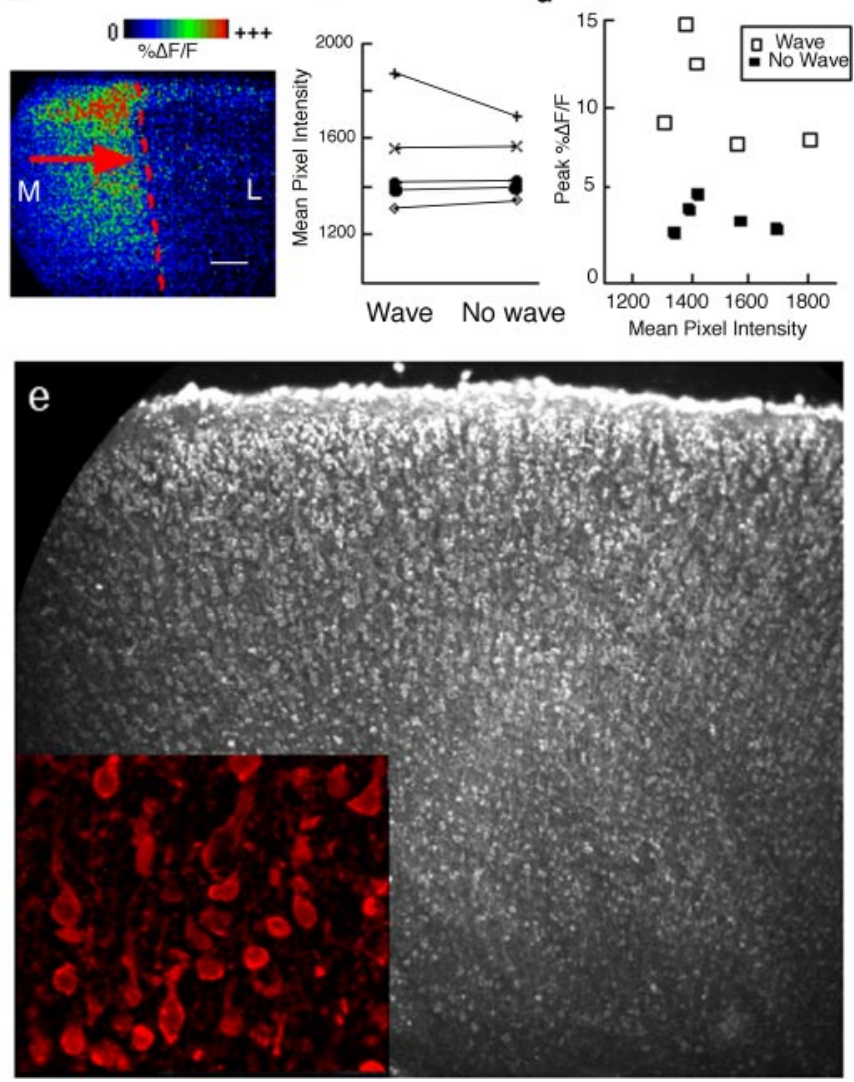

Figure 3. Cell density and fura-2 loading are similar in areas that sustain wave activity and those that do not. $\boldsymbol{a}$, Sample image of a fura-2-stained P4 slice illustrates the method used to assess cell density and dye loading in two neighboring subregions in the dorsal part of a coronal slice. Traces from the corresponding color-coded rectangular regions show the response of each region to bath-applied agonist. Note the absence of any obvious cytoarchitectonic boundary at the wave termination site. $\boldsymbol{b}$, Processed image highlighting the extent of the area that responded to agonist with a wave of activity in the region shown in $\boldsymbol{a}$. c, Values of mean pixel intensity for pairs of regions from five slices (P3-P4). $\boldsymbol{d}$, Peak $\% \Delta F / F$ as a function of the mean pixel intensity for each of the 10 regions shown in c shows that differences in the amplitude of the response are not accounted for by differences in dye loading or cell density, both of which contribute to mean pixel intensity. $\boldsymbol{e}$, Niss--stained section from the same region as in $\boldsymbol{a}$ also shows that no clear cytoarchitectonic discontinuity is present at the site of wave termination. M, Medial; L, lateral. The red arrows show the direction of wave propagation. Scale bar, $200 \mu \mathrm{m}$. The insets in $\boldsymbol{a}$ and e show $6 \times$ higher magnification of stained cells with fura- 2 and Nissl stain, respectively. 
a
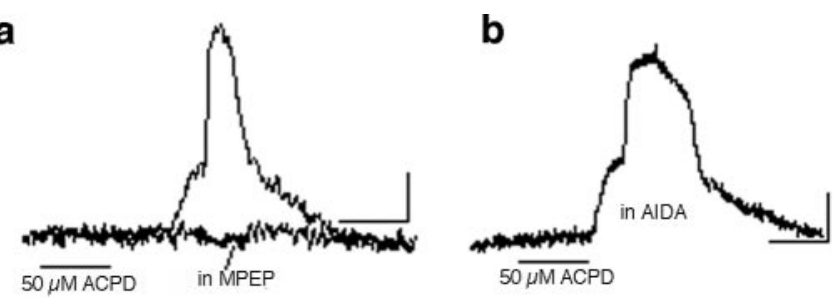

c
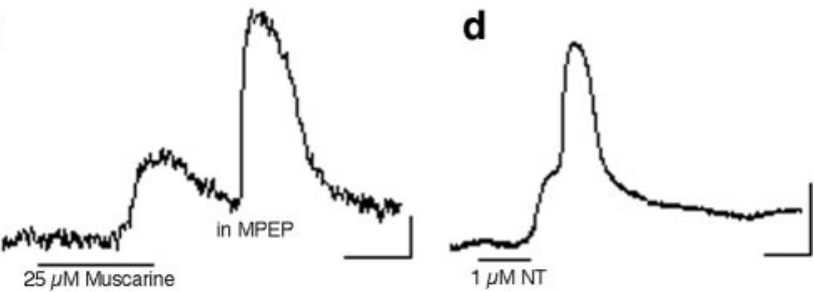

Figure 4. 0ther $\mathrm{Gq} / 11$-linked neurotransmitter receptors also trigger wave-like activation. The response to the group I/II mGluR agonist ACPD in the frontal cortex $(\boldsymbol{a})$ is indistinguishable from the response to muscarine. The ACPD effect is blocked by the mGluR5 antagonist MPEP ( 2.5 $\mu \mathrm{m} ; n=3)(\boldsymbol{a})$ but not by the mGluR1 antagonist AIDA $(200 \mu \mathrm{m} ; n=3)(\boldsymbol{b}) . \boldsymbol{c}$, In the presence of MPEP, a muscarinic response can still be obtained, indicating that the more abundant glutamatergic system is not responsible for indirectly mediating the effect induced by muscarine. $\boldsymbol{d}$, Activation of neurotensin (NT) receptors with neurotensin 8-13 also activates a synchronized network response. All records shown are from $\mathrm{P} 4-\mathrm{P} 5$ slices. Calibration: 5\% $\Delta F / F, 20 \mathrm{~s}$.

work activation, we considered whether it is glutamate, possibly released in response to stimulation of muscarinic receptors on cortical neurons, that was responsible for mediating, via mGluR5 receptors, the muscarinic effect on network activation. To test for this, we exposed slices to muscarine as in previous experiments, except that in this case, the mGluR5 antagonist MPEP was present in the bath. Despite the block of mGluR5, muscarine was able to induce activation normally (Fig. $4 c$ ), suggesting that stimulation of both glutamate and acetylcholine receptors is a parallel mechanism for triggering the large calcium responses seen in cortical networks at this age.

Another neurotransmitter receptor known to signal through G- $\alpha_{\mathrm{q} / 11}$ is the neurotensin receptor NTS1 (Vincent et al., 1999). Both the peptide neurotensin and its receptor are highly expressed during early postnatal cortical development (Hara et al., 1982; Palacios et al., 1988; Lepee-Lorgeoux et al., 1999). Experiments were performed using either neurotensin 8-13 (an active fragment of the tridecapeptide neurotensin, made from amino acids $8-13$ ) or the neurotensin-like peptide Neuromedin-N. Both peptides $(1 \mu \mathrm{M})$ were able to generate the signature network calcium signal associated with the propagating component of the response as seen with muscarine and ACPD (Fig. $4 d$ ).

As previously shown for muscarinic stimulation (Peinado, 2000), stimulation of neurotensin and mGluR5 receptors only generated wave-like activation until P7 but not later (data not shown).

\section{The network response to activation of Gq-coupled receptors occurs in other cortical areas}

Our inability to find any cytoarchitectonic features that correlated with the wave-competent area in the dorsal cortex precludes the unambiguous identification of this area by anatomical criteria. Its location, however, suggested that it might include area Fr2 (also known as the medial agranular cortex), a frontal area that extends caudally along the dorsal aspect of each hemisphere, near the midline. This prompted us to examine the frontal cortex more generally as well as other cortical regions. Responses ob- tained in the frontal pole in sagittal slices were similar to those obtained in the dorsal region of coronal slices [i.e., they included a sudden large amplitude component (the wave) riding on a more sustained, low-amplitude signal) (Fig. 5a). To compare the frontal and occipital cortex, we cut sagittal slices that included the entire cortex, from the anterior to posterior pole ( $n=10$ slices), and found that responses in the occipital (presumptive visual) cortex (Fig. 5b) resembled those described for more lateral regions of the cortex (presumed somatosensory) in coronal slices (i.e., only a low-amplitude response was observed).

Two other regions were identified that exhibited a wave-like response. First, in a subset of horizontal slices $(n=15)$ in which we examined the posterior half of the slice, we observed large agonist-triggered wave-like responses in an unidentified region of the temporal cortex that may, based on its location, correspond to the perirhinal cortex (Fig. 5b). A second area was identified in coronal slices ( $n=9$ slices) by examining the more ventral aspect of the lateral cortex, a region that, based on its location, should include or correspond to the insular cortex. This association area also generated large responses similar to those in the frontal cortex (Fig. 5c). In summary, the largest single region where waves can be triggered with agonist is in the frontal pole, but more posterior cortical areas along the rhinal sulcus as well as a medial dorsal strip also exhibit this behavior.

Wave-like responses to agonist involve a sudden switch from random to synchronous activity among participating neurons The feature that differentiates the population calcium signal in high-responding cortical areas from that in low-responding areas is not simply the amplitude of the calcium transient but, more interestingly, its rate of rise. To better understand the reasons for this difference, we analyzed responses and examined the time course of calcium transients in local groups of cells at a magnification that allowed imaging of individual cells $(n=5$ sagittal slices; four pups). This analysis revealed that in the frontal cortex, the rapid rate of rise of the calcium signal reflects a very tightly synchronized response onset among local neurons (Fig. 6a). In contrast, neurons in the occipital cortex respond with a lesser degree of temporal coordination and therefore fail to generate the fast rise in the population calcium signal that is the signature of the propagating wave. The maximum rate of rise in the fluorescent signals recorded in the frontal cortex is, on average, more than three times faster than in the occipital cortex (slope, $1.81 \pm$ 0.31 vs $0.64 \pm 0.19 ; p=0.005$ ) (Fig. $6 b$ ). Interestingly, the initial response of a randomly selected group of cells in the frontal cortex to application of muscarine is typically also highly disorganized, spread out over tens of seconds as in the occipital cortex (Fig. 6c). It is typically only later that the synchronized calcium transient is generated and travels as a wave throughout the network. How this transition from disorganized to coordinated activity comes about and why it only occurs in some cortical areas is still not clear. The fact that the disorganized part of the response to Gq-coupled receptor stimulation is seen in all areas, however, suggests that the mechanisms responsible for coordinating the wave-like activity involve something other than the response to agonist per se.

\section{$\mathrm{D}_{1}$ dopamine receptors modulate the response to activation of other receptors}

In light of the strong presence of waves in the frontal cortex, we were interested in examining the role of dopamine, a neurotransmitter commonly associated with frontal cortex function. Of the dopamine receptor subtypes, only $\mathrm{D}_{1}$ receptors are known to 


\section{a Frontal Ctx}
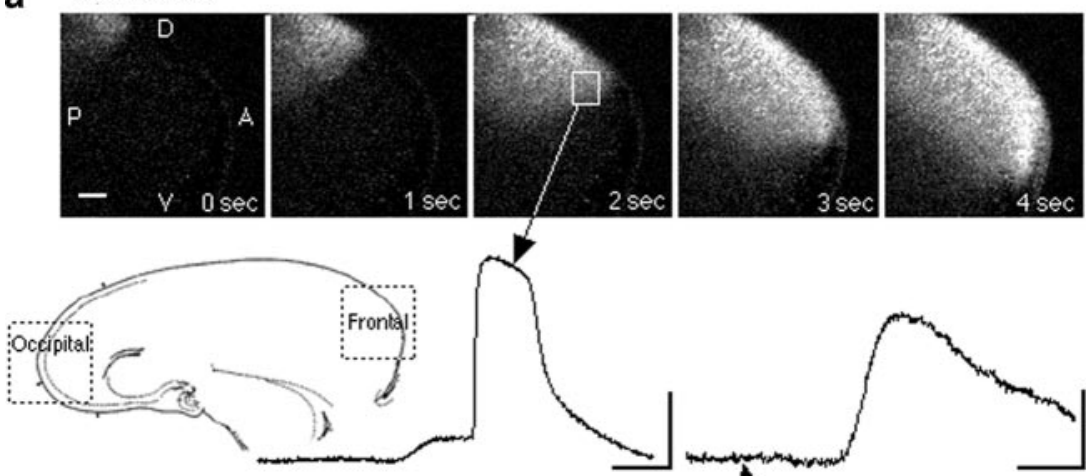

,

Occipital Ctx
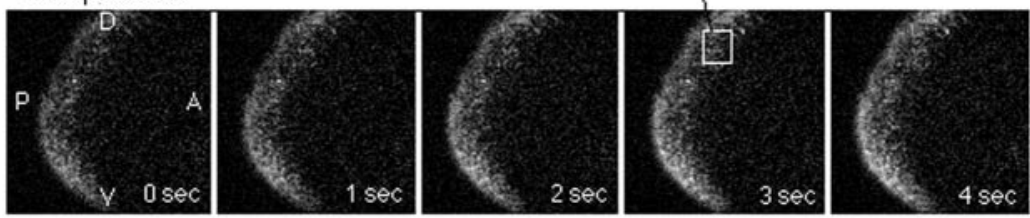

b
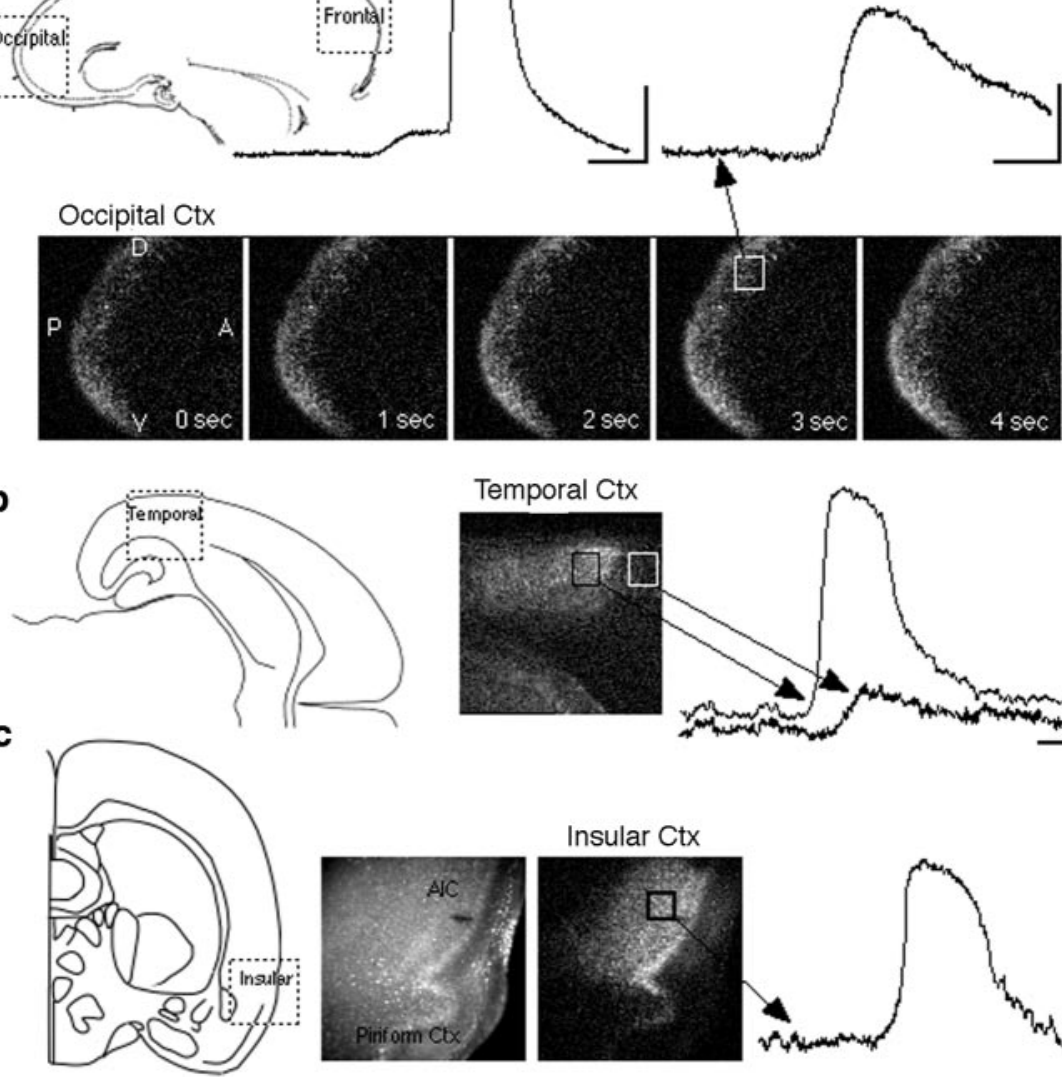

Figure 5. Examples from four additional cortical areas illustrate the dual nature of network responses to $\mathrm{Gq} / 11$-linked receptor stimulation. $\boldsymbol{a}$, Example of the differential response in two areas, frontal and occipital, obtained in single sagittal P5 slices containing the entire anteroposterior extent of one hemisphere. Processed image sequences (described in Materials and Methods) illustrate the spatiotemporal characteristics of the response at $1 \mathrm{~s}$ intervals during the peak of the response in each area. In the occipital cortex (Ctx), the response occurs diffusely throughout the area; in the frontal cortex, in addition to the low-level diffuse response (not visible in the processed images), there is a wave of strong activation that propagates horizontally across the cortex. Scale bar, $200 \mu \mathrm{m} . \boldsymbol{b}$, A response in the temporal cortex in a P4 horizontal slice. The trace derived from the white square shows response in a neighboring area presumed to be the parietal cortex. $c$, Example of a response in a P3 coronal slice recorded in the ventrolateral region, an area that contains the agranular insular cortex (AIC). The left image shows the raw fura- 2 fluorescence; the right image is processed (see Materials and Methods) to highlight the spatial extent of the response at its peak. Calibration: $5 \% \Delta F / F, 20$ s.

couple with G- $\alpha_{\text {q/11 }}$ (Undie and Friedman, 1990; Wang et al., 1995). However, $\mathrm{D}_{1}$ receptors couple not only with $\mathrm{Gq} / 11$ but to Gs proteins as well, in the latter case to activate adenylate cyclase (Wang et al., 1995; Lezcano et al., 2000). Because both Gq/11 and Gs are expressed in the immature cortex (Ihnatovych et al., 2002), it was unclear whether stimulation of $D_{1}$ receptors should result in a response similar to that elicited by the muscarinic, mGluR, and neurotensin receptors. Our initial attempts to elicit a response using the $\mathrm{D}_{1}$ agonist dihydrexidine were not successful. To look for a possible synergistic effect of $\mathrm{D}_{1}$ activation in conjunction with muscarinic activation, we reduced the concentration of muscarine to a subthreshold level $(6 \mu \mathrm{M})$, one that did not elicit a full response (Fig. $7 a$ ). We next tested the response to a

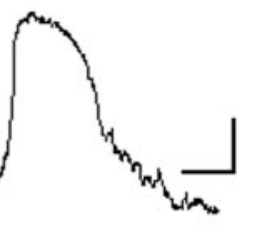

commonly used concentration $(40 \mu \mathrm{M})$ of the $\mathrm{D}_{1}$ agonist at the same cortical site (Fig. 7b). Finally, we lowered the concentration of dihydrexidine to $10 \mu \mathrm{M}$ and applied it briefly in combination with the subthreshold dose of muscarine. As shown in Figure $7 c$, the combined application of the two agonists produced a fullblown response in the cortical network. Additional evidence for a modulatory role of dopamine receptors in the response to other Gq/11-linked receptors comes from experiments in which ACPD was used as the stimulus. We took advantage of the fact that occasionally slices will not generate waves in response to ACPD even in areas that typically generate them. We noticed that in instances in which this was the case, it was possible to obtain full-blown responses to ACPD after bath application of dihydrexidine $(10 \mu \mathrm{M})$ for $10 \mathrm{~min}$ (three of four slices; data not shown).

The dopaminergic innervation of the cortex develops very early (Schmidt et al., 1982; Kalsbeek et al., 1988). Cortical slices prepared from rat pups between $\mathrm{P} 4$ and $\mathrm{P} 7$ would be expected to contain a substantial innervation by dopaminergic fibers and therefore possibly a background level of dopamine release and tonic activation of dopamine receptors, as has been reported in striatal slices (Aosaki et al., 1998). To determine whether the activation pattern elicited through Gq-linked receptors is dependent on tonic activation of $\mathrm{D}_{1}$ dopamine receptors, we performed experiments in which we treated slices continuously with the $\mathrm{D}_{1}$ antagonist SCH23390 $(10-20 \mu \mathrm{M})$. A $20 \mathrm{~s}$ application of the mGluR agonist ACPD was performed on these slices as in previous experiments to induce a wave of activation in the frontal cortex. We found that the $\mathrm{D}_{1}$ antagonist impaired the ability of ACPD to elicit a network response (Fig. $7 d$ ), suggesting that tonic activation is indeed present and necessary for wave generation. It is clear, however, that dopaminergic function does not constitute a sufficient determinant for wave-like activity, because the dopaminergic innervation continues to increase and is present in the adult cortex, whereas the waves cannot be induced by any of the three agonists after the first week. One possibility is that some component of the wave mechanism that is influenced by dopaminergic activity ceases to be present after the first week. Thus, the precise way in which dopamine enables wave-like activity during this period remains to be elucidated.

\section{Spontaneous wave-like activation in the frontal cortex}

The fact that multiple transmitters, including glutamate through mGluR5, can result in wave-like activation of the frontal cortex suggested that it might be possible to record waves occurring spontaneously. To find out whether spontaneous waves do in- 
a
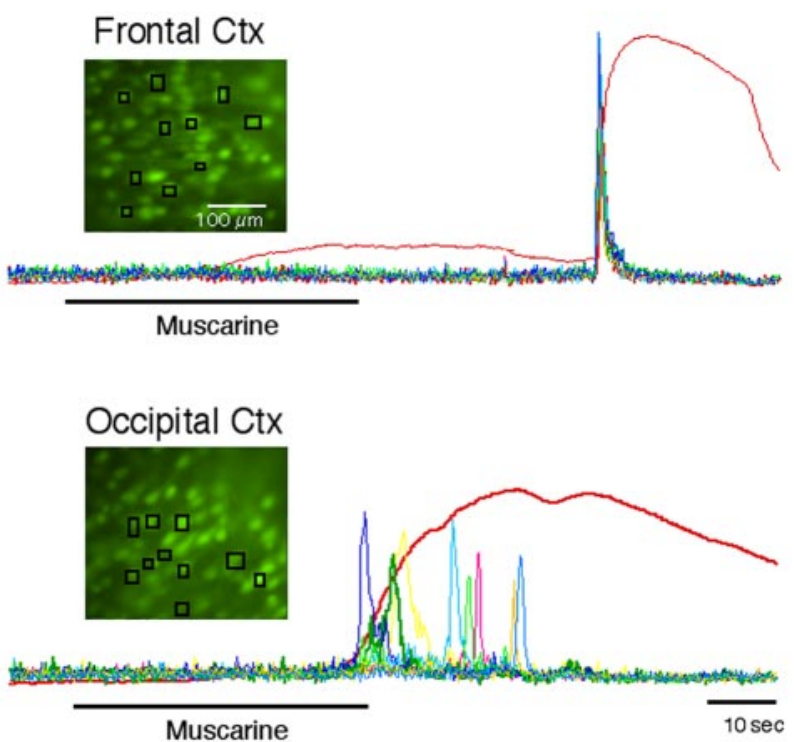

b

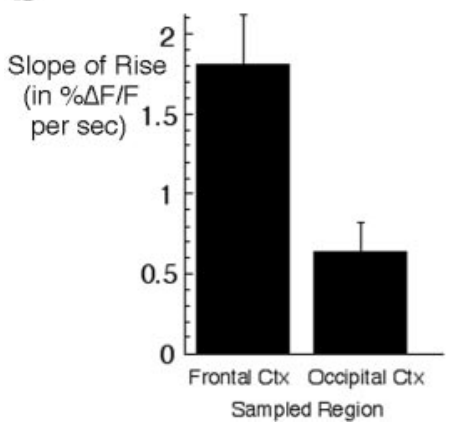

c

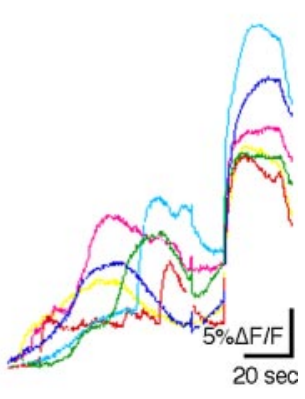

Figure 6. Synchronized responses among neighboring neurons underlies the sudden, largeamplitude calcium signal in the frontal cortex (Ctx). $\boldsymbol{a}$, Image sequences captured at high magnification were used to analyze the responses of individual neurons after muscarine (25 $\mu \mathrm{M})$ application in P4-P7 slices. Images show the location of the multiple small regions from which cell responses were sampled. The red traces show the response time course averaged for all of the cells in the field of view. Other colored traces show the first derivative of the response for each of the cells sampled. The peak of each first derivative trace identifies the time of maximal rate of increase in $\left[\mathrm{Ca}^{2+}\right]_{\mathrm{i}}$. Note that the fast-rising signal in the frontal cortex is associated with single-cell responses that are synchronized, whereas the slower signal in the occipital cortex is attributable to variable timing in the response of single cells. $\boldsymbol{b}$, Quantification of the difference in signal slope ( $\% \Delta F / F$ per second) in frontal and occipital areas ( $n=11$ and 10 , respectively) reveals a threefold faster rise time in the frontal cortex $(p<0.006)$. $c$, The synchronized response of cells in the frontal cortex is usually preceded by smaller nonsynchronous responses. These nonsynchronous responses make up the slow, low-amplitude component in the population (spatially averaged) signals shown in all other figures.

deed occur, we performed a set of experiments in the frontal cortex in which calcium imaging was done in the absence of any agonist stimulation. Twenty-seven slices prepared from animals between $\mathrm{P} 0$ and $\mathrm{P} 7$ were examined using repeated 12-min-long recordings at low magnification in an effort to detect spontaneous activation. In four of the slices, no wave-like activity was observed. In the remaining 23 slices (85\%), which included all ages examined, one or more waves were recorded. In all, 204 spontaneous waves were recorded in slices never exposed to exogenous agonist. The appearance of these waves in terms of the spatial propagation (Fig. $8 a$ ) and their signature waveform (Fig. $8 b$ ) differed from agonist-induced waves only in that spontaneous waves generally lack the prewave component of the response typical of the agonist-triggered waves.
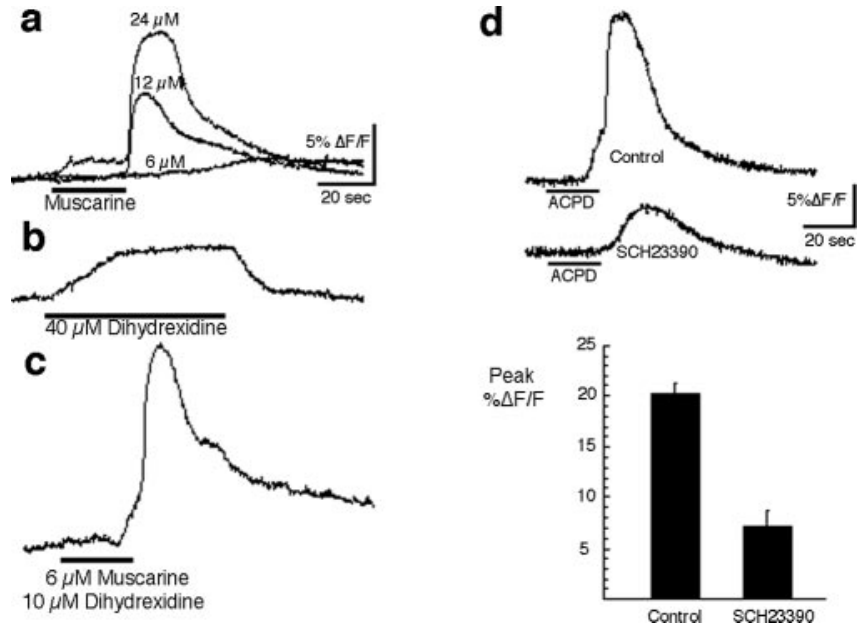

Figure 7. Dopamine $D_{1}$ receptors gate the activation induced through $\mathrm{Gq} / 11$-linked receptors. $\boldsymbol{a}$, A subthreshold dose of muscarine was obtained by progressively lowering the muscarine concentration until the fast, large-amplitude component of the response could not be induced. $\boldsymbol{b}$, A typical concentration of the $\mathrm{D}_{1}$ agonist dihydrexidine $(40 \mu \mathrm{m})$ was then applied to the slice, and the response at the same site was recorded. The change in fluorescence recorded during perfusion of $40 \mu \mathrm{m}$ dihydrexidine is probably a result of absorption by this compound at the fura-2 excitation or emission wavelengths, not because of a real calcium increase. c, Application of a reduced dihydrexidine concentration $(10 \mu \mathrm{m})$ together with a subthreshold dose of muscarine acts synergistically with the subthreshold muscarine stimulus to induce strong network activation (P5 slice). $\boldsymbol{d}$, Application of the $\mathrm{D}_{1}$ antagonist SCH23390 to the bath results in elimination of most of the response, suggesting that there is a basal level of $D_{1}$ receptor stimulation in slices and that this is necessary for the response ( $n=4$ slices; P4 -P5).

The most noticeable feature of spontaneous waves is how infrequently they occur. Although our data are derived from repeated 12 min records interrupted by brief periods during which data were stored, we can estimate the average interval between waves to be $\sim 27.9 \pm 12 \mathrm{~min}$ (mean $\pm \mathrm{SD}$ ). Figure $8 \mathrm{c}$ shows the distribution of intervals between successive waves obtained from 21 slices in which two or more waves were recorded. The data as presented assume that no events occurred in the periods between recordings, an assumption that may not be true in every instance. However, six intervals that appeared to be outliers (i.e., were $>2$ SDs away from the mean) and may have been the result of missed events were not included. At any rate, it is clear from the data presented that there is much variability in the duration of the intervals between events. Moreover, our calculated mean may well be an underestimate of the true average interval because we have excluded from our analysis the open-ended intervals, namely those occurring before the first recorded spontaneous wave and those occurring after the last recorded wave for each slice.

Because intervals between spontaneous events can be unpredictably long, it may not always be feasible to probe their pharmacology to the same extent as with agonist-induced waves. So far, however, based on their strikingly similar overall appearance, it seems that spontaneous and agonist-induced waves are one and the same phenomenon. A further indication of this is that the lateral boundary of wave propagation in the frontal cortex is similar for spontaneous and agonist-induced waves. In both cases, horizontal slices obtained from a plane dorsal to the rhinal sulcus show that there is little, if any, propagation into the lateral (presumptive parietal) cortex (Fig. $8 d$ ). On the other hand, slices cut more ventral, which include the cortex surrounding the rhinal sulcus, typically show propagation extending much further 

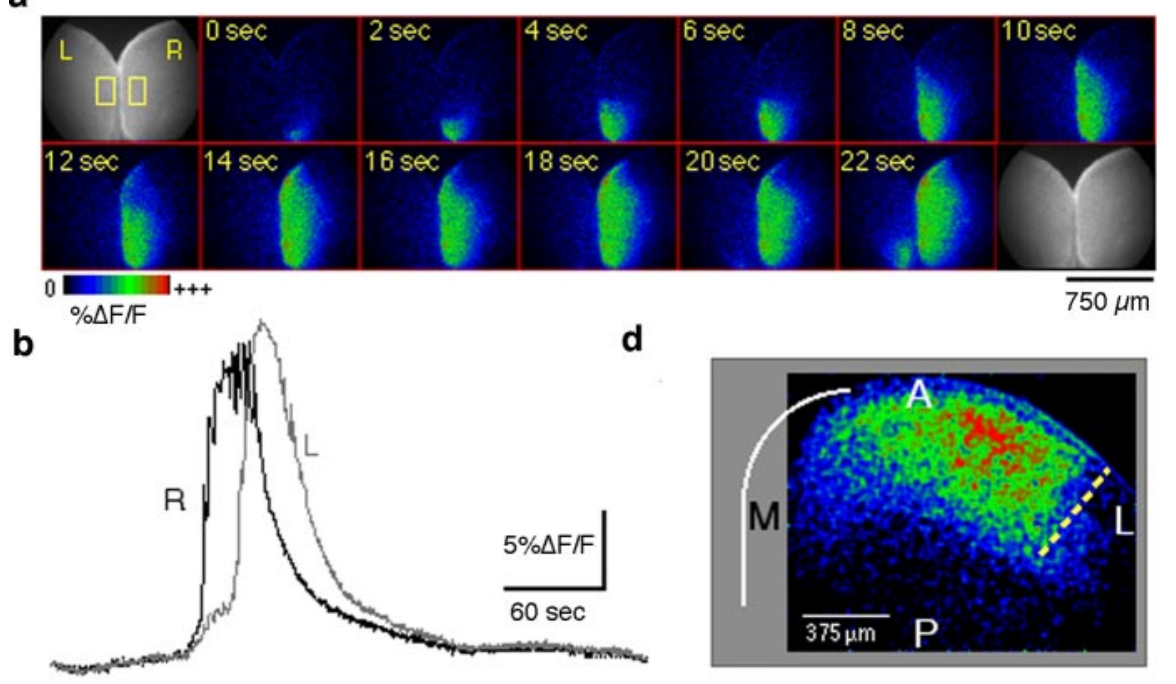

C

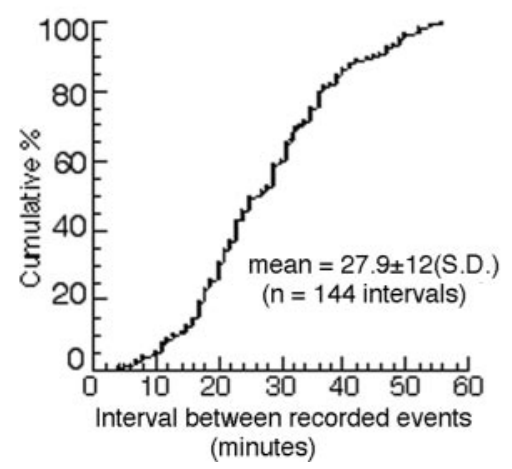

e

$(3.6 \%$

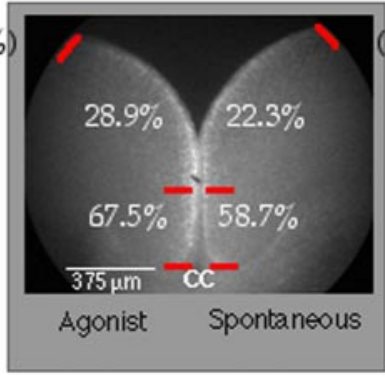

Figure 8. Spontaneous waves occur at low frequency in the frontal cortex in the absence of exogenous agonist. $\boldsymbol{a}$, Example of a spontaneous wave in a horizontal P4 slice. The sequence of processed images shows activity propagation starting in the medial cortex of the hemisphere labeled "R," near the CC (not visible). Only the first $22 \mathrm{~s}$ of this wave are shown. A wave in the hemisphere labeled " $\mathrm{L}$ " begins $\sim 20$ s after the one in R. $\boldsymbol{b}$, Traces from each of the two rectangular regions depicted on the first frame of $\boldsymbol{a}$ show the time course of the calcium signal. c, Data summarizing interevent intervals as inferred from multiple $12 \mathrm{~min}$ recordings on each of 23 slices. $d$, Extent of propagation of a spontaneous wave in the frontal cortex showing the lateral boundary (dotted line) in a P5 slice. A, Anterior; P, posterior; L, lateral; $M$, medial. e, Summary of initiation sites. The numbers refer to the percentage of waves initiating in each marked segment of the frontal cortex for agonist-induced (left) and spontaneous (right) waves recorded in different sets of slices. Waves originating outside the field of view are shown in parentheses.

caudally along the lateral cortex (data not shown). Both of these results are consistent with results obtained in coronal slices (described above) and indicate that: (1) the wave-competent region of the insular cortex seen in coronal slices is contiguous with the wave-competent region in the frontal cortex; and (2) spontaneous and induced waves behave similarly as far as the cortical regions they invade.

Another similarity concerns their site of initiation. We compared the sites of wave initiation for spontaneous and agonistinduced waves in the frontal region of P3-P5 horizontal slices. Our results show that there can be multiple sites of initiation in the frontal cortex regardless of how waves are triggered. In both cases, however, we found that a majority of waves $(58.7 \%$ of spontaneous, $67.5 \%$ of agonist-induced) arise from the most medial region of the cortex, a region that we defined (to adjust for age-related differences in brain size) as extending from the corpus callosum (CC) to the midpoint between the CC and the point at which the two hemispheres separate (Fig. 8a,e). This small medialmost region of the frontal cortex, extending an average of $665 \mu \mathrm{m}$ along the cortical surface (range, $418-798 \mu \mathrm{m}$ ), repre-
$(19.0 \%)$

sents a hot spot for initiation of both spontaneous and induced waves. The almost identical initiation profile is therefore another indication of the mechanistic similarity of the two types of wave.

The 27.9 min average interval of spontaneous waves is significantly longer than we might expect, given our ability to trigger waves with agonist as often as every 10 min. This raises two intriguing and related questions about spontaneous waves for which we currently have no answer: first, what triggers a spontaneous wave? Second, if global activation of Gq-coupled receptors is a prerequisite for wave generation, as our experiments seem to indicate, how do neurons throughout large expanses of the cortex in a slice become simultaneously capable of engaging in wave-like activity? Or, in other words, how does simultaneous activation of their Gq-coupled receptors come about in an isolated cortical slice absent the bathapplied agonist? One possibility is that there is always a basal level of receptor activation present that is sufficient to enable wave propagation and that all that is needed to trigger the wave therefore is a sudden local excitation. Our attempts to test this by focal electrical stimulation of the hot spot region have yielded results that do not support this "basal stimulation" hypothesis: despite clear evidence of intense local activation around the electrode, there is no propagation beyond the stimulation site unless agonist is applied exogenously (unpublished observations) [but see Peinado and Calderon (2002) for a similar experiment in the presumptive Fr2 region]. An alternative possibility is suggested by the studies of Dale et al. (2001) showing agonist-induced cyclical coupling and uncoupling of mGluR5 and mGluR1 to Gq. Under this scenario, a slow periodic oscillation in the effectiveness of Gq-coupled receptors driven by low levels of glutamate in the extracellular space would result in a waxing and waning of the probability of occurrence of spontaneous waves. Electrical stimulation would result in a wave if and only if it coincided with a peak in this hypothetical effectiveness cycle. At present, however, the possibility of such a scenario remains to be investigated.

\section{Discussion}

We have examined the spatiotemporal dynamics of calcium transients in immature cortical networks from different cortical areas, particularly in response to stimulation of specific metabotropic receptors. To accomplish this, we have taken advantage of the ability to examine the physiology of relatively large expanses of the cortex using fura- 2 imaging in slices to obtain a wide sampling of areas throughout the cortex. This approach initially revealed that an intense, highly coordinated network response could occur after brief stimulation of muscarinic receptors in some cortical areas. The most extensive region exhibiting this 
type of response turns out to be the frontal cortex. However, we also found other smaller regions that, strictly on the basis of their location, mapped to the temporal (putative perirhinal) cortex and insular cortex.

We have also shown that this form of network activity occurs in response to several different modulatory neurotransmitters. Our previous work (Peinado, 2000) had used only muscarinic agonists and showed that the M1 subtype, a receptor coupled predominantly with the Gq/11 G-protein isoform, was responsible for the effect. Here, we show that there are redundant pathways, involving other Gq/11-coupled receptors, capable of eliciting the same type of response. Thus, after brief applications of a ligand that signals through one or more of these receptors, immature cortical networks exhibit one of two forms of behavior depending on the cortical area examined. In some areas (e.g., parietal and occipital), the response consists of activity that when examined at high magnification is not tightly coordinated temporally, even among neighboring cells. This type of response is essentially identical to that previously described in the developing parietal cortex in response to stimulation of muscarinic and metabotropic glutamate receptors (Flint et al., 1999). In contrast to that type of response, imaging in other areas, most notably the frontal cortex, revealed activation that occurs in a temporally coordinated manner with large calcium transients occurring in most or all neurons in what appears to be a single explosive burst of activity that propagates as a wave over large cortical distances. This same network behavior was found to occur spontaneously in slices when long fura-2 recordings where done in the frontal cortex.

The network activation obtained spontaneously and in response to stimulation of $\mathrm{Gq} / 11$-linked receptors appears different in several respects from the cortical early network oscillations (cENOs) described in a study by Garaschuk et al. (2000). An important difference between their study and this one is that their study focused on the more frequent spontaneous activity originating in the entorhinal cortex. Spontaneous activity in the medial frontal cortex was either not recorded or not reported. A few cENOs were reported in regions anterior to the entorhinal cortex in slices prepared before P4. It is unclear, however, to what extent the pharmacological analysis included the waves originating in the anterior cortex, which, as they report, occur very infrequently. Our own work (unpublished observations) suggests that entorhinal cENOs, which occasionally propagate beyond the entorhinal cortex, are different, mechanistically and in time course, from the waves in the frontal cortex described here.

One major mechanistic difference is that the waves studied here persist in the presence of ionotropic glutamate receptor antagonists (Peinado, 2000), whereas the entorhinal activity disappears in the presence of the AMPA receptor antagonist CNQX (Garaschuk et al., 2000). The two types of waves, however, share at least one feature in common, namely their lack of dependence on GABAergic neurotransmission. Thus, the finding that picrotoxin does not block waves rules out a mechanism similar to the giant depolarizing potentials previously described in hippocampal networks during the neonatal period, a form of spontaneous activity that relies on the depolarizing action of GABA at these ages (Ben-Ari et al., 1989).

The lack of dependence on fast glutamatergic or GABAergic neurotransmission raises the question of how the waves described here propagate through the cortex. Propagation via gap junctions or some other type of signaling between glia (Parri et al., 2001) appears unlikely. Although some contribution from glial calcium to the overall calcium signal measured during waves cannot be excluded, a major role for glia in these waves is inconsistent with the finding that the wave component of the response is blocked by the sodium channel blocker tetrodotoxin (Peinado, 2000) and with the fact that cortical glia do not appear to express group I mGluRs (Lopez-Bendito et al., 2002). If gap junction coupling is involved, it is therefore more likely to be in the form of coupling between neurons (Peinado, 2001).

A caveat of both the study by Garaschuk et al. (2000) and the present study, indeed of all studies of the neonatal rodent cortex, is that anatomically the functional cortical areas can be defined even less precisely at these ages than in the mature cortex. A few area boundaries, such as that between the entorhinal and perirhinal cortices at the rhinal sulcus, can be identified visually, but this is not generally the case. Consequently, although we speculate based on topographical location that area Fr2, for example, is involved in our analysis on the dorsal region of coronal slices, we cannot know whether all of Fr2 is involved or to what extent neighboring motor areas (Fr1, M1) are activated as well, particularly in cases in which waves extend more laterally. In the absence of clear cytoarchitectonic boundaries, the mediolateral extent of wave propagation cannot be linked unambiguously to functional area boundaries. Variability in the location of area boundaries, however, could be one factor contributing to the variability in the lateral extent of wave propagation. For example, area boundaries are known to vary between individual animals (Zilles, 1985). Moreover, in the case of area Fr2 specifically, the lateral boundary does not run parallel to the midline (Zilles, 1985; Zilles and Wree, 1995) such that the lateral extent of Fr2 can increase threefold in going from a slice obtained from the midsection of the brain to one cut more rostral. Additionally, brain size is increasing quite rapidly at this age. Notwithstanding these uncertainties, our results indicate that network responses in the frontal and temporal cortex, broadly defined, are markedly different from cENOs originating in the entorhinal cortex and from Gq-mediated responses in occipital and parietal areas. In the latter areas, although agonist-induced activation occurs, it fails to achieve tight coordination between cells.

Why the developing cortex may require different forms of activity in different cortical areas is not clear. One possibility is that cortical areas that receive fewer direct sensory inputs, as might be the case for limbic areas, depend more on spontaneous forms of coordinated activity. A hint that the areas expressing wave-like responses might indeed be mostly limbic cortical areas is suggested by studies describing the distribution of the limbic system-associated membrane protein in the developing cortex (Horton and Levitt, 1988; Pimenta et al., 1996). Indeed, considering its presence in the frontal and perirhinal cortex and its absence in visual and somatosensory regions, the distribution of this protein overlaps with the cortical areas in which we have observed wave-like activation better than any other marker we are aware of. Whether the protein itself is in any way involved in generating the wave-like response or whether it is merely a marker that colocalizes with this type of response is not presently known.

Elucidating the molecular and cellular mechanisms underlying the tendency of select cortical areas to translate activation of Gq/11-linked receptors into a strong, coordinated network event that includes large calcium waves will require much additional work. The underlying difference may involve any of a number of components along the cascade leading to excitation as well as differences in the emergent properties of each network. We have shown that differences in the level of inhibition through $\mathrm{GABA}_{\mathrm{A}}$ 
receptors is not likely to fully explain the different network responses obtained in different cortical areas. Differences in the level of expression or function of mGlu, muscarinic, and neurotensinergic receptors in different cortical areas could be another explanation for the difference between high and low response areas. To our knowledge, however, expression patterns of these receptors in the developing cortex bear no particular resemblance to the topographic distribution of wave-like responses described here, receptors being mostly uniformly expressed throughout the cortex. Another possibility, for which our results provide support, is that it is the presence or absence of a second, synergistic pathway that determines the type of response in a particular cortical area. We have shown that simultaneous activation of dopamine receptors may be required for the large-amplitude, coordinated network response to occur. The heterogeneous distribution of dopaminergic afferents and dopamine receptors (Divac et al., 1978; Verney et al., 1982; Descarries et al., 1987; Gaspar et al., 1995), generally higher in the frontal and temporal cortex, may therefore be at least partly responsible for differences in the responses obtained in different cortical areas. However, although the role of dopamine is significant in that it may explain the regional expression of wave-like activity, it is clear that the mere presence of dopamine signaling in the cortex does not constitute a sufficient determinant for wave-like activity: the dopaminergic innervation continues to increase and is present in the adult cortex, whereas wave-like activity cannot be induced by any of the three agonists after the first week.

Another scenario that could influence the type of response is if in areas that exhibit wave responses the neurons themselves exhibit some intrinsic physiology or circuitry that specifically enables this type of network response. Under this scenario, dopamine might act to activate or facilitate this feature of neuronal physiology. A candidate for such a property is the ability of neurons to fire in a sustained manner. Sustained firing has been shown in vivo to take place in the absence of external cues and is thought to underlie the ability of higher cortical areas to hold information in working (short-term) memory (for review, see Durstewitz et al., 2000). As with the early wave-like network activation described here, it is unclear to what extent sustained firing in mature neurons can be a network property and to what extent it can be a cellular one (Marder et al., 1996; Aksay et al., 2001). Although the phenomenon of sustained firing has not been examined in the developing cortex, similarities between sustained firing in mature neurons and the phenomenon described in the present study raise the possibility that sustained firing may already be operating in a subset of immature cortical areas early on and may underlie the ability of these areas to generate waves. For example, in the mature cortex, sustained firing occurs preferentially in neurons of frontal and other higher associational areas; sustained firing is also modulated by dopamine, and it is also induced by muscarinic receptor activation (Sawaguchi and Goldman-Rakic, 1991; Broersen et al., 1994; Goldman-Rakic, 1995; Egorov et al., 2002). Thus, as mentioned above, it is still unclear why wave-like activation occurs during a brief developmental window. One possibility is that some component of the circuitry responsible for the wave-like activation is expressed transiently in these cortical areas. Another possibility is that some inhibitory mechanism sets in approximately at the end of the first week; yet another is that Gq-coupled signaling is activated more potently during early postnatal development (Dudek and Bear, 1989; Bevilacqua et al., 1995; Reid et al., 1996).
A final issue raised by our findings concerns the influence this form of activation might have on cortical development. Appropriate levels of neuronal activity, in terms of an optimal frequency of calcium transients, may be necessary for neurons to acquire their mature physiological properties (Ghosh and Greenberg, 1995; Zhang and Poo, 2001; Spitzer, 2002). Abnormal activation may therefore cause long-term changes in neuronal physiology as well as connectivity. In general, an abnormal level of activity, if it occurs at a time when the maturation of neuronal phenotype and connectivity are sensitive to activity, can be expected to significantly and possibly irreversibly alter the normal course of development and the long-term functional properties of networks, as have been shown to be the case in sensory pathways during "critical" periods (Wiesel, 1982; Goodman and Shatz, 1993; Katz and Shatz, 1996; Crair, 1999; Sur et al., 1999).

In light of the redundant pathways capable of triggering this form of network activity, and the finding that it occurs spontaneously in brain slices, it is likely that it occurs in vivo as well, although it is still unclear how often or under what circumstances. It is possible that expression of this large-scale activity in vivo is dependent on perturbations, including gene-environment interactions, not frequently encountered during normal development. In vivo recordings in unanesthetized pups will be required to clarify these points. In any case, the precedents for this form of activity affecting circuit formation and gene expression together with the uniqueness of the frontal cortex in terms of its interconnectedness to diverse brain regions suggest that further understanding the regulation and cellular mechanisms of this activity will provide important insights into the normal and abnormal development of multiple brain circuits and the higher-order behaviors they mediate.

\section{References}

Aksay E, Gamkrelidze G, Seung HS, Baker R, Tank DW (2001) In vivo intracellular recording and perturbation of persistent activity in a neural integrator. Nat Neurosci 4:184-193.

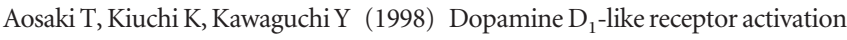
excites rat striatal large aspiny neurons in vitro. J Neurosci 18:5180-5190.

Ben-Ari Y (2001) Developing networks play a similar melody. Trends Neurosci 24:353-360.

Ben-Ari Y, Cherubini E, Corradetti R, Gaiarsa JL (1989) Giant synaptic potentials in immature rat CA3 hippocampal neurones. J Physiol (Lond) 416:303-325.

Bevilacqua JA, Downes CP, Lowenstein PR (1995) Transiently selective activation of phosphoinositide turnover in layer $\mathrm{V}$ pyramidal neurons after specific mGluRs stimulation in rat somatosensory cortex during early postnatal development. J Neurosci 15:7916-7928.

Borodinsky LN, Root CM, Cronin JA, Sann SB, Gu X, Spitzer NC (2004) Activity-dependent homeostatic specification of transmitter expression in embryonic neurons. Nature 429:523-530.

Broersen LM, Heinsbroek RP, de Bruin JP, Joosten RN, van Hest A, Olivier B (1994) Effects of local application of dopaminergic drugs into the dorsal part of the medial prefrontal cortex of rats in a delayed matching to position task: comparison with local cholinergic blockade. Brain Res 645:113-122.

Chub N, O’Donovan MJ (1998) Blockade and recovery of spontaneous rhythmic activity after application of neurotransmitter antagonists to spinal networks of the chick embryo. J Neurosci 18:294-306.

Crair MC (1999) Neuronal activity during development: permissive or instructive? Curr Opin Neurobiol 9:88-93.

Dale LB, Babwah AV, Bhattacharya M, Kelvin DJ, Ferguson SS (2001) Spatial-temporal patterning of metabotropic glutamate receptormediated inositol 1,4,5-triphosphate, calcium, and protein kinase $\mathrm{C}$ oscillations: protein kinase $\mathrm{C}$-dependent receptor phosphorylation is not required. J Biol Chem 38:35900-35908. 
De Felipe J, Marco P, Fairen A, Jones EG (1997) Inhibitory synaptogenesis in mouse somatosensory cortex. Cereb Cortex 7:619-634.

Descarries L, Lemay B, Doucet G, Berger B (1987) Regional and laminar density of the dopamine innervation in adult rat cerebral cortex. Neuroscience 21:807-824.

Divac I, Bjorklund A, Lindvall O, Passingham RE (1978) Converging projections from the mediodorsal thalamic nucleus and mesencephalic dopaminergic neurons to the neocortex in three species. J Comp Neurol 180:59-71.

Dudek SM, Bowen WD, Bear MF (1989) Postnatal changes in glutamate stimulated phosphoinositide turnover in rat neocortical synaptoneurosomes. Dev Brain Res 47:123-128.

Durstewitz D, Seamans JK, Sejnowski TJ (2000) Neurocomputational models of working memory. Nat Neurosci [Suppl] 3:1184-1191.

Egorov AV, Hamam BN, Fransen E, Hasselmo ME, Alonso AA (2002) Graded persistent activity in entorhinal cortex neurons. Nature 420:173-178.

Feller MB (1999) Spontaneous correlated activity in developing neural circuits. Neuron 22:653-656.

Flint AC, Dammerman RS, Kriegstein AR (1999) Endogenous activation of metabotropic glutamate receptors in neocortical development causes neuronal calcium oscillations. Proc Natl Acad Sci USA 96:12144-12149.

Garaschuk O, Hanse E, Konnerth A (1998) Developmental profile and synaptic origin of early network oscillations in the CA1 region of rat neonatal hippocampus. J Physiol (Lond) 507:219-236.

Garaschuk O, Linn J, Eilers J, Konnerth A (2000) Large-scale oscillatory calcium waves in the immature cortex. Nat Neurosci 3:452-459.

Gaspar P, Bloch B, Le Moine C (1995) D1 and D2 receptor gene expression in the rat frontal cortex: cellular localization in different classes of efferent neurons. Eur J Neurosci 7:1050-1063.

Ghosh A, Greenberg ME (1995) Calcium signaling in neurons: molecular mechanisms and cellular consequences. Science 268:239-247.

Goldman-Rakic PS (1995) Cellular basis of working memory. Neuron 14:477-485.

Goodman CS, Shatz CJ (1993) Developmental mechanisms that generate precise patterns of neuronal connectivity. Cell [Suppl] 72:77-98.

Gu X, Spitzer NC (1995) Distinct aspects of neuronal differentiation encoded by frequency of spontaneous $\mathrm{Ca}^{2+}$ transients. Nature 375:784-787.

Hara Y, Shiosaka S, Senba E, Sakanaka M, Inagaki S, Takagi H, Kawai Y, Takatsuki K, Matsuzaki T, Tohyama M (1982) Ontogeny of the neurotensin-containing neuron system of the rat: immunohistochemical analysis. I. Forebrain and diencephalon. J Comp Neurol 208:177-195.

Horton HL, Levitt P (1988) A unique membrane protein is expressed on early developing limbic system axons and cortical targets. J Neurosci 8:4653-4661.

Ihnatovych I, Novotny J, Haugvicova R, Bourova L, Mares P, Svoboda P (2002) Ontogenetic development of the G protein-mediated adenylyl cyclase signalling in rat brain. Brain Res Dev Brain Res 133:69-75.

Kalsbeek A, Voorn P, Buijs RM, Pool CW, Uylings HB (1988) Development of the dopaminergic innervation in the prefrontal cortex of the rat. J Comp Neurol 269:58-72.

Katz LC, Shatz CJ (1996) Synaptic activity and the construction of cortical circuits. Science 274:1133-1138.

Kilb W, Luhmann HJ (2003) Carbachol-induced network oscillations in the intact cerebral cortex of the newborn rat. Cereb Cortex 13:409-421.

Leinekugel X, Khazipov R, Cannon R, Hirase H, Ben-Ari Y, Buzsaki G (2002) Correlated bursts of activity in the neonatal hippocampus in vivo. Science 296:2049-2052.

Lepee-Lorgeoux I, Betancur C, Rostene W, Pelaprat D (1999) Differential ontogenetic patterns of levocabastine-sensitive neurotensin NT2 receptors and of NT1 receptors in the rat brain revealed by in situ hybridization. Brain Res Dev Brain Res 113:115-131.

Lezcano N, Mrzljak L, Eubanks S, Levenson R, Goldman-Rakic P, Bergson C (2000) Dual signaling regulated by calcyon, a D1 dopamine receptor interacting protein. Science 287:1660-1664.

Lopez-Bendito G, Shigemoto R, Fairen A, Lujan R (2002) Differential distribution of group I metabotropic glutamate receptors during rat cortical development. Cereb Cortex 12:625-638.

Luhmann HJ, Prince DA (1991) Postnatal maturation of the GABAergic system in rat neocortex. J Neurophysiol 65:247-263.

MacGregor DG, Chesler M, Rice ME (2001) HEPES prevents edema in rat brain slices. Neurosci Lett 303:141-144.

Marder E, Abbott LF, Turrigiano GG, Liu Z, Golowasch J (1996) Memory from the dynamics of intrinsic membrane currents. Proc Natl Acad Sci USA 93:13481-13486.

Meister M, Wong RO, Baylor DA, Shatz CJ (1991) Synchronous bursts of action potentials in ganglion cells of the developing mammalian retina. Science 252:939-943.

Milner LD, Landmesser LT (1999) Cholinergic and GABAergic inputs drive patterned spontaneous motoneuron activity before target contact. J Neurosci 19:3007-3022.

Moroni F, Lombardi G, Thomsen C, Leonardi P, Attucci S, Peruginelli F, Torregrossa SA, Pellegrini-Giampietro DE, Luneia R, Pellicciari R (1997) Pharmacological characterization of 1-aminoindan-1,5-dicarboxylic acid, a potent mGluR1 antagonist. J Pharmacol Exp Ther 281:721-729.

O'Donovan MJ (1999) The origin of spontaneous activity in developing networks of the vertebrate nervous system. Curr Opin Neurobiol 9:94-104.

Ongur D, Price JL (2000) The organization of networks within the orbital and medial prefrontal cortex of rats, monkeys and man. Cereb Cortex 10:206-219.

Owens DF, Boyce LH, Davis MB, Kriegstein AR (1996) Excitatory GABA responses in embryonic and neonatal cortical slices demonstrated by gramicidin perforated-patch recordings and calcium imaging. J Neurosci 16:6414-6423.

Palacios JM, Pazos A, Dietl MM, Schlumpf M, Lichtensteiger W (1988) The ontogeny of brain neurotensin receptors studied by autoradiography. Neuroscience 25:307-317.

Parri HR, Gould TM, Crunelli V (2001) Spontaneous astrocytic $\mathrm{Ca}^{2+}$ oscillations in situ drive NMDAR-mediated neuronal excitation. Nat Neurosci 4:803-812.

Paxinos G, Tork I, Tecott LH, Valentino KL (1991) Atlas of the developing rat brain. New York: Academic.

Peinado A (2000) Traveling slow waves of neural activity: a novel form of network activity in developing neocortex. J Neurosci 20:RC54(1-6).

Peinado A (2001) Immature neocortical neurons exist as extensive syncitial networks linked by dendrodendritic electrical connections. J Neurophysiol 85:620-629.

Peinado A, Calderon DP (2002) Hyperactivation of developing cortical circuits by acetylcholine and the ontogeny of abnormal cognition and emotion: findings and hypothesis. Prog Brain Res 145:131-142.

Penn AA, Riquelme PA, Feller MB, Shatz CJ (1998) Competition in retinogeniculate patterning driven by spontaneous activity. Science 279:2108-2112.

Pimenta AF, Reinoso BS, Levitt P (1996) Expression of the mRNAs encoding the limbic system-associated membrane protein (LAMP): II. Fetal rat brain. J Comp Neurol 375:289-302.

Reid SN, Daw NW, Gregory DS, Flavin H (1996) cAMP levels increased by activation of metabotropic glutamate receptors correlate with visual plasticity. J Neurosci 16:7619-7626.

Sanchez-Vives MV, McCormick DA (2000) Cellular and network mechanisms of rhythmic recurrent activity in neocortex. Nat Neurosci 10:1027-1034.

Sawaguchi T, Goldman-Rakic PS (1991) D1 dopamine receptors in prefrontal cortex: involvement in working memory. Science 251:947-950.

Schmidt RH, Bjorklund A, Lindvall O, Loren I (1982) Prefrontal cortex: dense dopaminergic input in the newborn rat. Brain Res 281:222-228.

Shatz CJ (1996) Emergence of order in visual system development. Proc Natl Acad Sci USA 93:602-608.

Spitzer NC (2002) Activity-dependent neuronal differentiation prior to synapse formation: the functions of calcium transients. J Physiol (Paris) 96:73-80

Spitzer NC, Debaca RC, Allen KA, Holliday J (1993) Calcium dependence of differentiation of GABA immunoreactivity in spinal neurons. J Comp Neurol 337:168-175.

Stead JDH, Neal CR, Evans SJ, Watson SJ, Akil H (2003) Variation in gene expression in the developing rat brain. Soc Neurosci Abstr 29:149.6.

Stellwagen D, Shatz CJ (2002) An instructive role for retinal waves in the development of retinogeniculate connectivity. Neuron 33:357-367.

Sur M, Angelucci A, Sharma J (1999) Rewiring cortex: the role of patterned activity in development and plasticity of neocortical circuits. J Neurobiol 41:33-43.

Undie AS, Friedman E (1990) Stimulation of a dopamine D1 receptor enhances inositol phosphates formation in rat brain. J Pharmacol Exp Ther 253:987-992.

Verney C, Berger B, Adrien J, Vigny A, Gay M (1982) Development of the dopaminergic innervation of the rat cerebral cortex. A light microscopic immunocytochemical study using anti-tyrosine hydroxylase antibodies. Brain Res 281:41-52. 
Vertes RP (2004) Differential projections of the infralimbic and prelimbic cortex in the rat. Synapse 51:32-58.

Vincent JP, Mazella J, Kitabgi P (1999) Neurotensin and neurotensin receptors. Trends Pharmacol Sci 20:302-309.

Wang HY, Undie AS, Friedman E (1995) Evidence for the coupling of Gq protein to D1-like dopamine sites in rat striatum: possible role in dopamine-mediated inositol phosphate formation. Mol Pharmacol 48:988-994.

Watt SD, Gu X, Smith RD, Spitzer NC (2000) Specific frequencies of spontaneous $\mathrm{Ca} 2+$ transients upregulate GAD 67 transcripts in embryonic spinal neurons. Mol Cell Neurosci 16:376-387.

Weliky M, Katz LC (1999) Correlational structure of spontaneous neuronal activity in the developing lateral geniculate nucleus in vivo. Science 285: 599-604.
Wiesel TN (1982) Postnatal development of the visual cortex and the influence of environment. Nature 299:583-591.

Wong RO (1999) Retinal waves and visual system development. Annu Rev Neurosci 22:29-47.

Yu CR, Power J, Barnea G, O’Donnell S, Brown HE, Osborne J, Axel R, Gogos JA (2004) Spontaneous neural activity is required for the establishment and maintenance of the olfactory sensory map. Neuron 42:553-566.

Yuste R, Peinado A, Katz LC (1992) Neuronal domains in developing neocortex. Science 257:665-669.

Zhang LI, Poo MM (2001) Electrical activity and development of neural circuits. Nat Neurosci [Suppl] 4:1207-1214.

Zilles K (1985) The cortex of the rat: a stereotaxic atlas. New York: Springer. Zilles K, Wree A (1995) Cortex: areal and laminar structure. In: The rat nervous system (Paxinos G, ed), pp 649-685. San Diego: Academic. 